Published in final edited form as:

Biochem Soc Trans. 2018 October 19; 46(5): 1191-1202. doi:10.1042/BST20170574.

\title{
Mammalian DNA methyltransferases: new discoveries and open questions
}

\author{
Humaira Gowher ${ }^{1}$ and Albert Jeltsch ${ }^{2}$ \\ ${ }_{1}^{1}$ Department of Biochemistry, Purdue University, West Lafayette, IN 47907, U.S.A. \\ ${ }^{2}$ Institute of Biochemistry and Technical Biochemistry, Department of Biochemistry, University of \\ Stuttgart, Stuttgart, Germany
}

\begin{abstract}
As part of the epigenetic network, DNA methylation is a major regulator of chromatin structure and function. In mammals, it mainly occurs at palindromic $\mathrm{CpG}$ sites, but asymmetric methylation at non-CpG sites is also observed. Three enzymes are involved in the generation and maintenance of DNA methylation patterns. DNMT1 has high preference for hemimethylated CpG sites, and DNMT3A and DNMT3B equally methylate unmethylated and hemimethylated DNA, and also introduce non-CpG methylation. Here, we review recent observations and novel insights into the structure and function of mammalian DNMTs (DNA methyltransferases), including new structures of DNMT1 and DNMT3A, data on their mechanism, regulation by post-translational modifications and on the function of DNMTs in cells. In addition, we present news findings regarding the allosteric regulation and targeting of DNMTs by chromatin modifications and chromatin proteins. In combination, the recent publications summarized here impressively illustrate the intensity of ongoing research in this field. They provide a deeper understanding of key mechanistic properties of DNMTs, but they also document still unsolved issues, which need to be addressed in future research.
\end{abstract}

\section{Introduction}

DNA methylation is a major regulator of chromatin structure in mammals. It is a component of the epigenome network and functions in concert with other epigenome modifications, most prominently histone tail modifications (review: [1,2]). In this review, we have compiled new insights into the structure and function of DNA methyltransferases (DNMTs) focusing on the mammalian enzymes. It is written as an update on earlier reviews on DNA methylation $[3,4]$ and the structure and mechanism of DNMTs [5,6]. The collection of recently published data provided here documents in an impressive way the intensity of ongoing research in this quite 'old' field.

In mammals, cytosine residues are methylated at levels between 3.5 and $4.5 \%$ in adult tissues depending on the cell type $[7,8]$. DNA methylation mainly occurs at palindromic

Correspondence: Albert Jeltsch (albert.jeltsch@ibtb.uni-stuttgart.de).

Competing Interests

The Authors declare that there are no competing interests associated with the manuscript. 
CpG sites, where it is present in both DNA strands (Figure 1), but asymmetric methylation at non-CpG sites is also observed (reviews: $[4,9,10]$ ). After replication, DNA methylation at CpG sites can be maintained by DNMT1, a maintenance methyltransferase, which has high preference for hemimethylated CpG sites. In contrast, two additional DNMTs, DNMT3A and DNMT3B, do not show preference for hemimethylated target sites. They are involved in the de novo generation of DNA methylation patterns during germ cell development and in the early embryonic phase. Unlike DNMT1, DNMT3A and DNMT3B also introduce non$\mathrm{CpG}$ methylation. Despite its overarching elegance, recent data show that this establishment and maintenance model of DNA methylation is too simplified and all DNMTs in concert with TET 5mC hydroxylases, which are involved in DNA demethylation, regulate DNA methylation levels through a dynamic equilibrium of site-specific gain and loss of methylation (review: [11]).

\section{Recent discoveries related to DNMT1 enzymes}

\section{Structure and mechanism of DNMT1}

Similar to other mammalian DNMTs, DNMT1 comprises a catalytic C-terminal and regulatory $\mathrm{N}$-terminal part (Figure 2A). The catalytic domain is conserved among all bacterial and eukaryotic DNA-(cytosine C5)-methyltransferases and all of them follow a common catalytic mechanism for transferring a methyl group from AdoMet to the target cytosine base (reviews: [5,6,12]) (Figure 2B). This mechanism involves target base flipping and binding of the cytosine into a catalytic pocket, hydrogen bonded to the side chain of a glutamate from a conserved ENV motif. The methylation reaction is initiated by the attack of a Cys residue from a conserved PCQ motif (PCN in the case of DNMT3 enzymes) on the C6-position of the aromatic ring, which is supported by acid/base catalysis of the glutamate. This step is followed by methyl group transfer to the C5-position and resolution of the covalent enzyme-DNA complex assisted by acid-base catalysis by an undefined base, perhaps a water molecule (reviews: $[5,12]$ ). The amino acid motifs containing these catalytic residues were identified in early sequence alignments and found to be conserved in all enzyme of this type (reviews: $[12,13])$. This general mechanism was recently confirmed in a kinetic isotope effect study with DNMT1, revealing that methyl transfer indeed occurs after the attack of the active site Cys residue and the methyl transfer reaction is the chemically rate-limiting step of the enzyme [14].

Different structural analyses of DNMT1 have provided strong evidence for an allosteric regulation of DNMT1 activity by autoinhibition and its interaction with chromatin modifications (review: [6]). The structure of DNMT1 in the absence of DNA showed that the RFTS domain binds to the catalytic domain occluding the DNA-binding site (Figure 3A). This indicated that DNMT1 must exist in at least two different conformations, a catalytically inactive state, in which the RFTS domain blocks the active site, and a catalytically active state, in which DNA is bound in the active site [15]. Dynamic autoinhibition by the RFTS domain was also observed in biochemical studies [16,17], further showing that weakening of RFTS domain binding to the catalytic domain increased the activity of DNMT1 [18]. 
The structure of a truncated DNMT1 containing the CXXC, BAH and catalytic domains showed that an unmethylated DNA specifically bound to the CXXC domain, but not to the catalytic domain [19]. Based on this observation, an additional autoinhibition model was proposed, in which binding of unmethylated CG sites to the CXXC domain would prevent their methylation, which was supported by kinetic data. However, the role of the CXXC domain in the specificity of DNMT1 is not yet clear, because another study showed that mutations in the CXXC domain that block its DNA binding did not change the preference of full-length DNMT1 for hemimethylated CpG sites [20].

The molecular interactions that confer specificity for hemimethylated $\mathrm{CpG}$ sites were revealed in the structure of a DNMT1 C-terminal fragment lacking the CXXC domain with hemimethylated DNA bound in a catalytically competent conformation [21] (Figure 3B). In this structure, DNMT1 forms several base-specific contacts with its target sequence at the methylated cytosine-guanine base pair, which were all shown to be essential for catalysis in a biochemical study [22]. The target cytosine is flipped out of the DNA helix and bound into a protein pocket, which presents the active site residues conserved among all DNA-(cytosine C5)-methyltransferases described above (Figure 3C). Side chains from the catalytic and recognition loops are inserted into the DNA through both grooves filling the space emptied by base flipping. The $5 \mathrm{mC}$ methyl group of the hemimethylated $\mathrm{CpG}$ site approaches a hydrophobic pocket formed by residues from the catalytic domain, which explains the preference of DNMT1 for hemimethylated sites. This study supports previous bio-chemical observations, which suggested that DNMT1 catalytic domain has an intrinsic specificity for hemimethylated DNA [20]. Additional conformational changes of the DNA were observed including flipping of another base, though these observations were not supported by biochemical studies [22].

Interestingly, the various structures of DNMT1 differ in the precise conformation of an ahelix following the active site loop, which either adopts a kinked (in inactive complexes) or straight conformation (in the complex of DNMT1 with hemimethylated DNA). A mutational study combined with MD simulations could identify two residues, mutations of which affected these conformational transitions and led to reduced catalytic activity [23] showing how dynamic conformational changes of DNMT1 are essential for catalysis.

\section{UHRF1 interaction of DNMT1}

UHRF1 belongs to the family of RING-finger type E3 ubiquitin ligases. The observations that UHFR1 co-localizes with DNMT1 and PCNA at replicating heterochromatic regions and that DNMT1 association with chromatin was lost in UHFR1 knock-out (KO) cells [24,25] were key discoveries, which stimulated many follow-up studies on the cross-talk between chromatin modifications, other chromatin factors and the activity of DNMT1. UHRF1 KO is embryonically lethal in mice and UHRF1-deficient embryos showed strongly reduced levels of genome-wide DNA methylation, indicating that UHRF1 has an essential role in the maintenance of DNA methylation $[24,25]$. These observations led to a model that UHFR1 recruits DNMT1 to replicated hemimethylated DNA to facilitate its efficient remethylation. The cooperation of PCNA and UHRF1 in DNMT1 targeting has been 
documented by showing that expression of a DNMT1-PCNA fusion protein can rescue DNA methylation in the absence of UHRF1 [26].

Like DNMT1, UHRF1 is a huge multidomain protein (review: [6]). The structural, mechanistic and functional details of the DNMT1-UHRF1 interaction have been subject of intense investigations showing a complex and multifaceted mechanism. UHRF1 stimulates the catalytic activity of DNMT1 by an interaction with the DNMT1 RFTS domain, which appears to open the autoinhibited conformation [17,27]. UHRF1 binds to hemimethylated DNA with its SET and RING-associated domain (review: [28]). Moreover, its tandem Tudor domain (TTD) and the plant homeodomain (PHD) bind H3K9me3 in a cooperative reaction $[29,30]$. The binding of UHRF1 to H3K9me3 is required for the localization of UHRF1 to heterochromatin and for the maintenance of DNA methylation, since a mutation in TTD, which prevents binding of UHRF1 to H3K9me3, abolished both functions [31,32]. However, most recent work showed that the knock-in of a UHRF1 gene with mutated H3K9me3binding site into uhfr1 deletion cells led to an almost complete recovery of DNA methylation, indicating that the H3K9me3-binding site of UHRF1 alone is not essential for DNA methylation [37]. Disruption of an H3R2-binding pocket in the PHD domain of UHRF1 (which is needed for H3 tail binding) abolished DNA methylation by DNMT1 in cells as well [33]. Recently, methylation of H3R2 by PRMT6, an enzyme that is often upregulated in cancer, was also shown to disrupt UHRF1 binding and maintenance DNA methylation [34]. Reductions in $\mathrm{H} 3 \mathrm{~K} 9 \mathrm{me} 2$ and in expression of UHRF1 are observed during global DNA demethylation when ESCs are transited from the serum-to-2i medium, suggesting a connection between both processes [35]. Regulation of global DNA methylation via the UHRF1-DNMT1 axis by Zscan4 has also been implicated in the control of telomere elongation [36].

The E3 ligase activity of the UHRF1 RING domain ubiquitinates H3 at K14, K18 and K23 [33,38-40]. A ubiquitin interaction motif was identified in the N-terminal part of DNMT1 and shown to be essential for DNA methylation in vivo [33]. Recently, two papers showed structures of the DNMT1 RFTS domain sand-wiched by two ubiquitin moieties from a dual ubiquitinated H3 tail (at K18 and K23) [39,40] (Figure 4A). In the context of full-length DNMT1, this binding must lead to a rearrangement of the RFTS domain, which could trigger the stimulation of DNMT1's activity, highlighting once more the central role of the RFTS domain-mediated autoinhibition in the regulation of DNMT1. Moreover, direct binding of the ubiquitin-like domain of UHRF1 to the same site in DNMT1 was also reported [40].

\section{Regulation of DNMT1 by PTMs}

In addition to ubiquitination, several other PTMs have been found on DNMT1 (review: [6]). For example, DNMT1 is monomethylated at K142 by SET7/9 mainly during late S-phase, which promotes proteasomal degradation of the methyltransferase in a cell cycle-dependent manner [41]. Very recently, it has been shown that the L3MBTL3 protein binds K142 methylated DNMT1 and triggers its ubiquitination and proteolysis [42].

The abundance of DNMT1 during cell cycle is also regulated by acetylation of the lysine residues in the GK linker of DNMT1 catalyzed by the acetyltransferase Tip60 [43,44]. GK 
acetylation leads to ubiquitination of DNMT1 by UHRF1, resulting in its proteasomal degradation at the end of DNA replication. In turn, histone deacetylase 1 increases the stability of DNMT1 [43,44]. This effect is meditated by the deacetylation of the GK linker, followed by binding of the USP7 deubiquitinase to DNMT1, which prevents its ubiquitination [45]. DNMT1 and USP7 form a stable, soluble complex that associates with UHRF1 as a trimeric complex on chromatin [46]. The crystal structure of a human DNMT1 fragment starting with the CXXC domain in complex with USP7 revealed that the interaction of both proteins occurs through the GK linker of DNMT1 and an acidic pocket near the C-terminus of USP7 [45]. Mutations of these acidic residues in USP7 and the absence of acetylation of the GK linker in DNMT1 disrupted the interaction between DNMT1 and USP7, leading to increased proteasomal degradation of DNMT1 [45]. Interestingly, binding of USP7 was also shown to increase the activity of DNMT1 in vitro [46]. However, recent data showed no change in DNMT1 levels in USP7 KO cells [47]. Furthermore, mutation of the GK repeat to GQ, which cannot be acetylated, did not have an effect on the stability and activity of DNMT1 in cells [47], thus refuting an essential role of USP7 and GK repeat acetylation in the regulation of DNMT1 activity and stability. It is unclear if these differences are due to the use of different cell types in these experiments, which is particularly important, given differences in the rate of proliferation and cell cycle control.

\section{Kinetics of maintenance methylation after DNA replication}

As mentioned above, DNMT1 is the main enzyme responsible for DNA methylation after DNA replication. DNMT1 contains a PCNA interaction region in its $\mathrm{N}$-terminal part, which is essential for its targeting to the replication fork [48]. Past studies have shown that deletion of the N-terminal region of DNMT1 including the PCNA interaction region creates a DNMT1 hypomorphic variant leading to reduced overall methylation levels in cells and delayed kinetics of remethylation [49]. BrdU pulse-chase experiments in HCT116 cells containing wild-type DNMT1 showed that remethylation was almost complete after a 30 min pulse with BrdU, but it was substantially delayed in cells with hypomorphic DNMT1 [49].

Recently, two papers studied the kinetics of remethylation after DNA replication using modified bisulfite conversion-based approaches coupled with deep sequencing. Charlton et al. [50] combined BrdU labeling and immunoprecipitation with genome-wide bisulfite sequencing and showed biphasic remethylation kinetics of nascent DNA. One fraction of methylation increased during the first hour after replication followed by a slower phase of up to $4 \mathrm{~h}$. Studies in DNMT KO cells revealed that the DNNT3 enzymes had a limited effect on post-replicative DNA methylation rates, but EZH2 sites with DNA methylation showed delayed remethylation, which was dependent on DNMT3 enzymes. Analysis of methylation patterns in individual DNA molecules revealed a processive reaction mechanism of DNMT1 in agreement with previous biochemical studies (review: [5]). Xu and Corces [51] used a similar approach, but found that the majority of remethylation had already occurred $20 \mathrm{~min}$ after DNA replication. In addition, they reported the discovery of $\sim 800$ stable hemimethylated $\mathrm{CpG}$ sites enriched at $\mathrm{CTCF} /$ cohesin sites, but this result was only based on 
bisulfite conversion coupled with deep sequencing and not validated with an independent technique.

\section{Recent discoveries related to DNMT3 enzymes}

\section{Structures and mechanism of DNMT3 enzymes}

The DNMT3 family comprises three orthologs, DNMT3A, DNMT3B and DNMT3L, all of which share considerable sequence similarity (Figure 2A). DNMT3L lacks catalytic activity, but it stimulates DNMT3A and DNMT3B and regulates their multimerization and nuclear localization (review: [6]). A DNMT3A/3L C-terminal domain structure published in 2007 showed the formation of a linear $3 \mathrm{~L}-3 \mathrm{~A}-3 \mathrm{~A}-3 \mathrm{~L}$ heterotetramer, which is mediated through two distinct interfaces, a 3A-3A central ' $\mathrm{RD}$ ' interface and two peripheral 3A-3L ' $\mathrm{FF}$ ' interfaces [52]. Additional work showed that all interfaces also mediate the self-interaction of DNMT3A (review: [6]). The linear heterotetramer contains two active sites (one in each DNMT3A subunit) in a distance corresponding to $8-10$ bp of DNA. Methylation studies confirmed the preference of DNMT3A to co-methylate two $\mathrm{CpG}$ sites placed at this distance [52,53]. Recently, 8-10 bp methylation patterns were also observed for non-CpG methylation catalyzed by DNMT3A in ES cells and neurons [54].

Different structures of a longer DNMT3A fragment containing the ADD chromatin interaction domain (see below) led to the discovery of an allosteric regulation of the catalytic activity of this enzyme [55] (Figure 4B). One structure revealed a conformation, in which the ADD domain interacts with the DNA-binding region of the catalytic domain, thus autoinhibiting its activity (inhibitory binding site). A second structure was generated in the presence of an $\mathrm{H} 3$ tail peptide bound to the ADD domain. It showed the ADD domain bound to the catalytic domain at another site, which should not interfere with enzyme activity (allosteric binding site). Interestingly, binding of the H3 peptide to the ADD domain occurs by interaction with residues, which are involved in the autoinhibitory-binding interface. Therefore, peptide binding is only possible in the active conformation and this conformation is stabilized in the presence of the H3 peptide. Enzyme kinetics confirmed that binding of the $\mathrm{H} 3$ tail peptide to the ADD domain leads to the release of autoinhibiton and an activation of DNMT3A [55], in agreement with biochemical studies published earlier [56,57] (review: $[6])$.

Recently, the first structures of DNMT3A/3L heterotetramers with DNA were published [58] (Figure 5A). In one of them, two short oligonucleotides were bound to the two active sites, in the second, one DNA containing two CpG sites in a distance of $14 \mathrm{bp}$ was used. The structures were obtained after the formation of covalent bonds between the active site Cys residues and modified bases introduced at the positions of the target cytosines. It is, therefore, unclear how the two site structure resembles a complex containing two $\mathrm{CpG}$ sites in $8-10$ bp distance as found to be preferentially methylated in biochemical studies (reviews: $[5,6])$. As in other DNMTs, the target cytosine is rotated out of the DNA helix and placed into the active site pocket, where it interacts with the catalytic resides described above (Figure 5B). The cavity after base flipping is filled by V716 from the target recognition loop. The guanine base of the $\mathrm{CpG}$ site is recognized by R836, the loss of which resulted in a reduced preference for $\mathrm{CpG}$ site methylation in vitro and in cells [58]. While the DNA 
bound to the two active sites was relaxed, the part of the DNA connecting them in the double-site substrate revealed a sharp bend accompanied by a strong compression of the major groove. Several residues make contacts with the bases flanking the $\mathrm{CpG}$ dinucleotide. This explains the strong flanking sequence preferences of DNMT3A, which have been previously observed (reviews: [5,6]). Moreover, the structure demonstrates that the R882 residue in human DNMT3A, which is frequently mutated in AML cancers (review: [59]), is involved in a backbone DNA contact. This observation can explain the strong shift in flanking sequence preference of the $\mathrm{R} 882 \mathrm{H}$ cancer mutation that was reported recently [60] and may contribute to the cancer-promoting effect of this mutation.

Recently, another unexpected activity of DNMTs was discovered by showing that different DNMTs (bacterial M.SssI and mouse DNMT3A catalytic domain) generate low levels of $3 \mathrm{mC}$ as a byproduct [61]. This modified base represents an alkylation damage of DNA, which in cells is repaired by ALKB2 family enzymes in an oxidative process. Indeed, it has been found that ALKB2 enzymes are evolutionarily strongly connected with active DNMTs in many species [61], highlighting the functional connection between them.

\section{Multimerization and processivity of DNMT3 enzymes}

As mentioned before, DNMT3A has two interfaces for hetero- and homomultimerization, and it can form dimers, tetramers and higher oligomers (review: [6]). Higher protein oligomers of DNMT3A can bind to more than one DNA strand oriented roughly in parallel [62], which could support binding of adjacent linker DNA regions in chromatin. Indeed, different studies recently showed a periodicity of co-methylation of $\mathrm{CpG}$ sites during de novo methylation in the range of $\sim 180$ nucleotides $[63,64]$, which roughly fits to the distance of two linker regions separated by a nucleosome, suggesting that a DNMT3 multimer could bind to and methylate adjacent linker DNA regions. A similar pattern was also observed in DNMT3 introduced non-CpG methylation [54]. Most residues in the two interfaces are conserved in DNMT3B, which is likely to form similar multimers, but this property has not yet been demonstrated. DNMT3L contains only one interface that restricts the DNMT3A/3L complex to a tetramer, which cannot oligomerize further [62]. Additionally, DNMT3A and DNMT3A/3L complexes multimerize on DNA next to each other [65]. In recent years, this multimerization has been shown to mediate cooperative DNA binding by DNMT3A [66] and to contribute to the efficient DNA methylation by DNMT3A in cells [67].

Interestingly, while DNMT3A binds cooperatively to DNA and formation of protein-DNA complexes prevents processive DNA methylation [66], recent work has demonstrated that DNMT3B does not bind to DNA cooperatively and it has a processive methylation mechanism [68], in agreement with an earlier study [69]. It was also shown that mutation of the arginine residue in DNMT3B analogous to R882 of DNMT3A had no effect on the catalytic activity or processivity of the DNMT3B enzyme. This study also suggests that the differences in the interface residues between DNMT3A and DNMT3B influence the distinct catalytic mechanisms of the two enzymes [68]. Future biochemical studies characterizing the interface residues of DNMT3B and/or its crystal structure analysis will be critical for understanding the distinct targets and biological functions of DNMT3A and DNMT3B. 
Moreover, both enzymes are known to interact and form mixed complexes [70], which adds another level of complexity to this question.

\section{Chromatin interaction of DNMT3 enzymes}

The N-terminal parts of DNMT3 enzymes contain two important chromatin interaction domains, the PWWP domain specific for binding of H3K36me3 and the ADD domain binding to the $\mathrm{N}$-terminus of the $\mathrm{H} 3$ tail (review: [6]). ADD binding to $\mathrm{H} 3$ is disrupted by methylation or acetylation of $\mathrm{K} 4$ or phosphorylation of T3 and T6 [56]. The functional role of the readout of these chromatin marks was studied experimentally by designing of DNMT3A ADD variants that were no longer sensitive towards K4 methylation or T6 phosphorylation [71]. Indeed, expression of these DNMT3A mutants in cells perturbed the differentiation program by causing defects in lineage commitment during ESC differentiation. However, the pluripotency and self-renewal genes in these cells were decreased similar to that in wild-type cells. Interestingly, another study showed a critical role of DNMT3A in methylation of pluripotency gene enhancers that is required for pluripotency gene repression during ESC differentiation. This study revealed a prominent role of the ADD domain in the targeting of DNMT3 enzymes at enhancers of pluripotency genes, where LSD1-dependent H3K4 demethylation was necessary for localized DNMT3A binding and activation, which led to enhancer methylation [72]. Taken together, these two studies demonstrate the biological relevance of epigenetic cross-talk mechanisms and their importance during development.

Recently, the structure the PWWP domain ( present in DNMT3A and DNMT3B) bound to an $\mathrm{H} 3$ peptide containing H3K36me3 was solved [73] and confirmed the binding pocket previously identified in biochemical studies [74]. Using DNMT3B KO mouse ES cell lines, intragenic DNA methylation was shown to be deposited by DNMT3B [63,75]. This activity of DNMT3B was dependent on SETD2 deposited H3K36me3 through the interaction of PWWP with H3K36 methylation. Gene body methylation by DNMT3B was shown to prevent spurious transcription initiation in line with the general function of gene body H3K36 methylation [76]. Another important role of H3K36me3 binding to the PWWP domain was described in a study showing that DNMT3A and DNMT3B associate with most active enhancers in epidermal stem cells in an H3K36me3-dependent manner, suggesting that this binding is mediated by the PWWP domain [77]. Both proteins differ in their effects on enhancer DNA modification. DNMT3B was involved in enhancer body methylation, while DNMT3A co-operates with TET2 promoting enhancer DNA hydroxymethylation. Interestingly, both DNMT3A and DNMT3B were required for enhancer activity and enhancer RNA production, illustrating a dual regulatory potential of DNA methylation for gene repression and activation.

\section{New genes and function of isoforms}

Recent studies have shown that the Dnmt $3 b$ gene in mice underwent a duplication forming $D n m t 3 c$, another active DNMT gene, which has a specific role in the silencing of retrotransposons in the development of male germ cells [78,79]. While it is clear that the human genome does not encode additional DNMTs, it will be interesting to see if similar 
careful inspections will also reveal more DNMT3 homologs in other mammalian subbranches.

In addition to gene duplications, there is potential for more functional specialization of DNMTs through the flexible use of alternative start sites and splicing isoforms. There are two known isoforms of DNMT3A, a longer DNMT3A1 and a shorter DNMT3A2, which is expressed from an internal start site [80]. It has been shown that DNMT3A2 is the dominant form in embryonic tissues, while DNMT3A1 is expressed in all tissues. Recent work revealed that the DNMT3A1 enzyme localizes to the shores of bivalent $\mathrm{CpG}$ island promoters, suggesting that the very N-terminal segment absent in DNMT3A2 interacts with other proteins and may have a role in targeting of DNMT3A1 [81].

For DNMT3B, several splicing isoforms have been identified, many of which are catalytically inactive due to deletions in the catalytic domain but some also contain deletions in the N-terminal part. Interestingly, one catalytically inactive splice variant (DNMT3B3) is the dominant isoform of DNMT3B expressed in somatic cells [82]. It was shown that inactive variants still interact with DNMT3A and DNMT3B, and they could regulate their catalytic activity [83]. Recently, it was shown that catalytically inactive DNMT3B isoforms could also bind to DNMT3A and stimulate its gene body methylation in somatic cells [84].

\section{Non-CpG methylation by DNMT3 enzymes}

Quantification and distribution of non-CpG methylation from genome-wide studies showed its enrichment in gene bodies and transposons at $>2 \%$ of cytosines in neurons, $>1 \%$ in frontal cortex and embryonic cells, while it is lower in most other cell types (review: [10]). Interestingly, non-CpG methylation in ES cells and in early development mainly occurs in a CAG context, while in differentiated neurons, CAC methylation is mainly observed (review: [10]).

Biochemical studies showed that non-CpG methylation can be introduced by DNMT3 enzymes, due to their relaxed specificity (reviews: [5,6]). However, DNMT1, the most active and important DNMT in mammals, shows an exclusive CpG specificity and is almost unable to methylate non-CpG sites [20]. Therefore, while $\mathrm{CpG}$ methylation is efficiently duplicated after each cell division, this is not true for non-CpG methylation, which must be generated de novo each time after DNA replication. Interestingly, although CNG sites are palindromic, DNA methylation at these sites is also not 'maintained' in the sense that in mammals no DNMT exists, which has specificity for hemimethylated CNG sites. Methylation analyses in DNMT KO cell lines clearly demonstrated that DNMT3 enzymes are responsible for nonCpG methylation [85]. Consequently, non-CpG methylation is mainly found in tissues with high expression of DNMT3 enzymes like embryonic tissues and neurons. In neurons particularly, the lack of cell division also favors the stable presence of non-CpG methylation. Studies in mouse showed that after re-expression of DNMT3A or DNMT3B in TKO ES cells, DNMT3A preferred introduction of CAC methylation, while DNMT3B was more active in CAG methylation [54]. This finding is in agreement with the general observation that DNMT3B has a role in early development and DNMT3A is highly expressed in brain tissues. In the brain, $\mathrm{CpG}$ and $\mathrm{CpA}$ methylation is observed in the gene bodies of long $\mathrm{MeCP} 2$ repressed genes [86]. Disruption of CpA methylation by conditional DNMT3A KO 
revealed that $\mathrm{CpA}$ methylation is critical for binding of $\mathrm{MeCP} 2$ and repression of long genes [87]. In another study, binding of MeCP2 to non-CpG methylated DNA was found to regulate gene expression in both directions (up and down) in adult mouse brain [88].

\title{
Outlook
}

The precise control of DNA methylation patterns is essential for human cells. This implies that the regulation of DNMT activity and stability as well as their recruitment to genomic target sites are very crucial processes. Past research has led to the emergence of common themes of DNMT control, like keeping DNMTs in an inactive autoinhibited ground state to avoid aberrant activity, or combined targeting and regulation of their catalytic activity. However, we are just starting to understand the intricate network of PTMs, chromatin modifications and chromatin factors, including non-coding RNAs, that governs DNMT regulation. Many details are still unclear and many more processes are likely to be still undiscovered, implying exciting times to come for DNMT research.

\author{
Abbreviations \\ DNMTs DNA methyltransferases \\ KO knock-out \\ PHD plant homeodomain \\ TTD tandem Tudor domain
}

\section{References}

1. Soshnev AA, Josefowicz SZ and Allis CD (2018) Greater than the sum of parts: complexity of the dynamic epigenome. Mol. Cell 69, 533 10.1016/j.molcel.2018.01.015

2. Allis CD and Jenuwein T (2016) The molecular hallmarks of epigenetic control. Nat. Rev. Genet 17, 487-500 10.1038/nrg.2016.59 [PubMed: 27346641]

3. Jones PA (2012) Functions of DNA methylation: islands, start sites, gene bodies and beyond. Nat. Rev. Genet 13, 484-492 10.1038/nrg3230 [PubMed: 22641018]

4. Schübeler D (2015) Function and information content of DNA methylation. Nature 517, 321-326 10.1038/nature14192 [PubMed: 25592537]

5. Jurkowska RZ, Jurkowski TP and Jeltsch A (2011) Structure and function of mammalian DNA methyltransferases. ChemBioChem 12, 206-222 10.1002/cbic.201000195 [PubMed: 21243710]

6. Jeltsch A and Jurkowska RZ (2016) Allosteric control of mammalian DNA methyltransferases - a new regulatory paradigm. Nucleic Acids Res 44, 8556-8575 10.1093/nar/gkw723 [PubMed: 27521372]

7. Globisch D, Münzel M, Müller M, Michalakis S, Wagner M, Koch S et al. (2010) Tissue distribution of 5-hydroxymethylcytosine and search for active demethylation intermediates. PLoS ONE 5, e15367 10.1371/journal.pone.0015367 [PubMed: 21203455]

8. Münzel M, Globisch D, Brückl T, Wagner M, Welzmiller V, Michalakis S et al. (2010) Quantification of the sixth DNA base hydroxymethylcytosine in the brain. Angew. Chem. Int. Ed. Engl 49, 5375-5377 10.1002/anie.201002033 [PubMed: 20583021]

9. Jeong M and Goodell MA (2014) New answers to old questions from genome-wide maps of DNA methylation in hematopoietic cells. Exp. Hematol 42, 609-617 10.1016/j.exphem.2014.04.008 [PubMed: 24993071] 
10. He Y and Ecker JR (2015) Non-CG methylation in the human genome. Annu. Rev. Genomics Hum. Genet 16, 55-77 10.1146/annurev-genom-090413-025437 [PubMed: 26077819]

11. Jeltsch A and Jurkowska RZ (2014) New concepts in DNA methylation. Trends Biochem. Sci 39, 310-318 10.1016/j.tibs.2014.05.002 [PubMed: 24947342]

12. Jeltsch A (2002) Beyond Watson and Crick: DNA methylation and molecular enzymology of DNA methyltransferases. ChemBioChem 3, 274-293 10.1002/1439-7633(20020402)3:4\&1t;274::AIDCBIC274\&gt;3.0.CO;2-S [PubMed: 11933228]

13. Cheng X (1995) Structure and function of DNA methyltransferases. Annu. Rev. Biophys. Biomol. Struct 24, 293-318 10.1146/annurev.bb.24.060195.001453 [PubMed: 7663118]

14. Du Q, Wang Z and Schramm VL (2016) Human DNMT1 transition state structure. Proc. Natl Acad. Sci. U.S.A 113, 2916-2621 10.1073/pnas.1522491113 [PubMed: 26929335]

15. Takeshita K, Suetake I, Yamashita E, Suga M, Narita H, Nakagawa A et al. (2011) Structural insight into maintenance methylation by mouse DNA methyltransferase 1 (Dnmt1). Proc. Natl Acad. Sci. U.S.A 108, 9055-9059 10.1073/pnas.1019629108 [PubMed: 21518897]

16. Syeda F, Fagan RL, Wean M, Avvakumov GV, Walker JR, Xue S et al. (2011) The replication focus targeting sequence (RFTS) domain is a DNA-competitive inhibitor of Dnmt1. J. Biol. Chem 286, 15344-15351 10.1074/jbc.M110.209882 [PubMed: 21389349]

17. Bashtrykov P, Jankevicius G, Jurkowska RZ, Ragozin S and Jeltsch A (2014) The UHRF1 protein stimulates the activity and specificity of the maintenance DNA methyltransferase DNMT1 by an allosteric mechanism. J. Biol. Chem 289, 4106-4115 10.1074/jbc.M113.528893 [PubMed: 24368767]

18. Bashtrykov P, Rajavelu A, Hackner B, Ragozin S, Carell T and Jeltsch A (2014) Targeted mutagenesis results in an activation of DNA methyltransferase 1 and confirms an autoinhibitory role of its RFTS domain. ChemBioChem 15, 743-748 10.1002/cbic.201300740 [PubMed: 24532244]

19. Song J, Rechkoblit O, Bestor TH and Patel DJ (2011) Structure of DNMT1-DNA complex reveals a role for autoinhibition in maintenance DNA methylation. Science 331, 1036-1040 10.1126/ science.1195380 [PubMed: 21163962]

20. Bashtrykov P, Jankevicius G, Smarandache A, Jurkowska RZ, Ragozin S and Jeltsch A (2012) Specificity of Dnmt1 for methylation of hemimethylated CpG sites resides in its catalytic domain. Chem. Biol 19, 572-578 10.1016/j.chembiol.2012.03.010 [PubMed: 22633409]

21. Song J, Teplova M, Ishibe-Murakami S and Patel DJ (2012) Structure-based mechanistic insights into DNMT1-mediated maintenance DNA methylation. Science 335, 709-712 10.1126/science. 1214453 [PubMed: 22323818]

22. Bashtrykov P, Ragozin S and Jeltsch A (2012) Mechanistic details of the DNA recognition by the Dnmt1 DNA methyltransferase. FEBS Lett 586, 1821-1823 10.1016/j.febslet.2012.05.026 [PubMed: 22641038]

23. Ye F, Kong X, Zhang H, Liu Y, Shao Z, Jin J et al. (2018) Biochemical studies and molecular dynamic simulations reveal the molecular basis of conformational changes in DNA methyltransferase-1. ACS Chem. Biol 13, 772-781 10.1021/acschembio.7b00890 [PubMed: 29381856]

24. Sharif J, Muto M, Takebayashi S.-i., Suetake I, Iwamatsu A, Endo TA et al. (2007) The SRA protein Np95 mediates epigenetic inheritance by recruiting Dnmt1 to methylated DNA. Nature 450, 908-912 10.1038/nature06397 [PubMed: 17994007]

25. Bostick M, Kim JK, Esteve P-O, Clark A, Pradhan S and Jacobsen SE (2007) UHRF1 plays a role in maintaining DNA methylation in mammalian cells. Science 317, 1760-1764 10.1126/science. 1147939 [PubMed: 17673620]

26. Liu X, Gao Q, Li P, Zhao Q, Zhang J, Li J et al. (2013) UHRF1 targets DNMT1 for DNA methylation through cooperative binding of hemi-methylated DNA and methylated H3K9. Nat. Commun 4, 1563 10.1038/ncomms2562 [PubMed: 23463006]

27. Berkyurek AC, Suetake I, Arita K, Takeshita K, Nakagawa A, Shirakawa M et al. (2014) The DNA methyltransferase Dnmt 1 directly interacts with the SET and RING finger-associated (SRA) domain of the multifunctional protein Uhrf1 to facilitate accession of the catalytic center to hemimethylated DNA. J. Biol. Chem 289, 379-386 10.1074/jbc.M113.523209 [PubMed: 24253042] 
28. Jeltsch A (2008) Reading and writing DNA methylation. Nat. Struct. Mol. Biol 15, 1003-1004 10.1038/nsmb1008-1003 [PubMed: 18836494]

29. Xie S, Jakoncic J and Qian C (2012) UHRF1 double tudor domain and the adjacent PHD finger act together to recognize K9me3-containing histone H3 tail. J. Mol. Biol 415, 318-328 10.1016/j.jmb. 2011.11.012 [PubMed: 22100450]

30. Rothbart SB, Dickson BM, Ong MS, Krajewski K, Houliston S, Kireev DB et al. (2013) Multivalent histone engagement by the linked tandem Tudor and PHD domains of UHRF1 is required for the epigenetic inheritance of DNA methylation. Genes Dev 27, 1288-1298 10.1101/ gad.220467.113 [PubMed: 23752590]

31. Nady N, Lemak A, Walker JR, Avvakumov GV, Kareta MS, Achour M et al. (2011) Recognition of multivalent histone states associated with heterochromatin by UHRF1 protein. J. Biol. Chem 286, 24300-24311 10.1074/jbc.M111.234104 [PubMed: 21489993]

32. Rothbart SB, Krajewski K, Nady N, Tempel W, Xue S, Badeaux AI et al. (2012) Association of UHRF1 with methylated H3K9 directs the maintenance of DNA methylation. Nat. Struct. Mol. Biol 19, 1155-1160 10.1038/nsmb.2391 [PubMed: 23022729]

33. Qin W, Wolf P, Liu N, Link S, Smets M, La Mastra F et al. (2015) DNA methylation requires a DNMT1 ubiquitin interacting motif (UIM) and histone ubiquitination. Cell Res 25, 911-929 10.1038/cr.2015.72 [PubMed: 26065575]

34. Veland N, Hardikar S, Zhong Y, Gayatri S, Dan J, Strahl BD et al. (2017) The arginine methyltransferase PRMT6 regulates DNA methylation and contributes to global DNA hypomethylation in cancer. Cell Rep 21, 3390-3397 10.1016/j.celrep.2017.11.082 [PubMed: 29262320]

35. von Meyenn F, Iurlaro M, Habibi E, Liu NQ, Salehzadeh-Yazdi A, Santos F et al. (2016) Impairment of DNA methylation maintenance is the main cause of global demethylation in naive embryonic stem cells. Mol. Cell 62, 983 10.1016/j.molcel.2016.06.005 [PubMed: 27315559]

36. Dan J, Rousseau P, Hardikar S, Veland N, Wong J, Autexier C et al. (2017) Zscan4 inhibits maintenance DNA methylation to facilitate telomere elongation in mouse embryonic stem cells. Cell Rep 20, 1936-1949 10.1016/j.celrep.2017.07.070 [PubMed: 28834755]

37. Zhao Q, Zhang J, Chen R, Wang L, Li B, Cheng H et al. (2016) Dissecting the precise role of H3K9 methylation in crosstalk with DNA maintenance methylation in mammals. Nat. Commun 7 , 12464 10.1038/ncomms12464 [PubMed: 27554592]

38. Nishiyama A, Yamaguchi L, Sharif J, Johmura Y, Kawamura T, Nakanishi K et al. (2013) Uhrf1dependent H3K23 ubiquitylation couples maintenance DNA methylation and replication. Nature 502, 249-253 10.1038/nature12488 [PubMed: 24013172]

39. Ishiyama S, Nishiyama A, Saeki Y, Moritsugu K, Morimoto D, Yamaguchi L et al. (2017) Structure of the Dnmt1 reader module complexed with a unique two-mono-ubiquitin mark on histone H3 reveals the basis for DNA methylation maintenance. Mol. Cell 68, 350-360.e7 10.1016/j.molcel.2017.09.037 [PubMed: 29053958]

40. Li T, Wang L, Du Y, Xie S, Yang X, Lian F et al. (2018) Structural and mechanistic insights into UHRF1-mediated DNMT1 activation in the maintenance DNA methylation. Nucleic Acids Res 46, 3218-3231 10.1093/nar/gky104 [PubMed: 29471350]

41. Esteve P-O, Chin HG, Benner J, Feehery GR, Samaranayake M, Horwitz GA et al. (2009) Regulation of DNMT1 stability through SET7-mediated lysine methylation in mammalian cells. Proc. Natl Acad. Sci. U.S.A 106, 5076-5081 10.1073/pnas.0810362106 [PubMed: 19282482]

42. Leng F, Yu J, Zhang C, Alejo S, Hoang N, Sun H et al. (2018) Methylated DNMT1 and E2F1 are targeted for proteolysis by L3MBTL3 and CRL4 ${ }^{\text {DCAF5 }}$ ubiquitin ligase. Nat. Commun 9, 1641 10.1038/s41467-018-04019-9 [PubMed: 29691401]

43. Du Z, Song J, Wang Y, Zhao Y, Guda K, Yang S et al. (2010) DNMT1 stability is regulated by proteins coordinating deubiquitination and acetylation-driven ubiquitination. Sci. Signal 3, ra80 10.1126/scisignal.2001462

44. Qin W, Leonhardt H and Spada F (2011) Usp7 and Uhrf1 control ubiquitination and stability of the maintenance DNA methyltransferase Dnmt1. J. Cell. Biochem 112, 439-444 10.1002/jcb.22998 [PubMed: 21268065] 
45. heng J, Yang H, Fang J, Ma L, Gong R, Wang P et al. (2015) Molecular mechanism for USP7mediated DNMT1 stabilization by acetylation. Nat. Commun 6, 7023 10.1038/ncomms8023 [PubMed: 25960197]

46. Felle M, Joppien S, Németh A, Diermeier S, Thalhammer V, Dobner T et al. (2011) The USP7/ Dnmt 1 complex stimulates the DNA methylation activity of Dnmt 1 and regulates the stability of UHRF1. Nucleic Acids Res 39, 8355-8365 10.1093/nar/gkr528 [PubMed: 21745816]

47. Yarychkivska O, Tavana O, Gu W and Bestor TH (2018) Independent functions of DNMT1 and USP7 at replication foci. Epigenet. Chromatin 11, 9 10.1186/s13072-018-0179-Z

48. Chuang LS-H, Ian H-I, Koh T-W, Ng H-H, Xu G and Li BFL (1997) Human DNA-(cytosine-5) methyltransferase-PCNA complex as a target for p2 $1^{\text {WAF1 }}$. Science 277, 1996-2000 10.1126/ science.277.5334.1996 [PubMed: 9302295]

49. Egger G, Jeong S, Escobar SG, Cortez CC, Li TWH, Saito Y et al. (2006) Identification of DNMT1 (DNA methyltransferase 1) hypomorphs in somatic knockouts suggests an essential role for DNMT1 in cell survival. Proc. Natl Acad. Sci. U.S.A 103, 14080-14085 10.1073/pnas. 0604602103 [PubMed: 16963560]

50. Charlton J, Downing TL, Smith ZD, Gu H, Clement K, Pop R et al. (2018) Global delay in nascent strand DNA methylation. Nat. Struct. Mol. Biol 25, 327-332 10.1038/s41594-018-0046-4 [PubMed: 29531288]

51. Xu C and Corces VG (2018) Nascent DNA methylome mapping reveals inheritance of hemimethylation at CTCF/cohesin sites. Science 359, 1166-1170 10.1126/science.aan5480 [PubMed: 29590048]

52. Jia D, Jurkowska RZ, Zhang X, Jeltsch A and Cheng X (2007) Structure of Dnmt3a bound to Dnmt3L suggests a model for de novo DNA methylation. Nature 449, 248-251 10.1038/ nature06146 [PubMed: 17713477]

53. Jurkowska RZ, Anspach N, Urbanke C, Jia D, Reinhardt R, Nellen W et al. (2008) Formation of nucleoprotein filaments by mammalian DNA methyltransferase Dnmt3a in complex with regulator Dnmt3L. Nucleic Acids Res 36, 6656-6663 10.1093/nar/gkn747 [PubMed: 18945701]

54. Lee J-H, Park S-J and Nakai K (2017) Differential landscape of non-CpG methylation in embryonic stem cells and neurons caused by DNMT3s. Sci. Rep 7, 11295 10.1038/ s41598-017-11800-1 [PubMed: 28900200]

55. Guo X, Wang L, Li J, Ding Z, Xiao J, Yin X et al. (2015) Structural insight into autoinhibition and histone H3-induced activation of DNMT3A. Nature 517, 640-644 10.1038/nature13899 [PubMed: 25383530]

56. Zhang Y, Jurkowska R, Soeroes S, Rajavelu A, Dhayalan A, Bock I et al. (2010) Chromatin methylation activity of Dnmt3a and Dnmt3a/3L is guided by interaction of the ADD domain with the histone H3 tail. Nucleic Acids Res 38, 4246-4253 10.1093/nar/gkq147 [PubMed: 20223770]

57. Li B-Z, Huang Z, Cui Q-Y, Song X-H, Du L, Jeltsch A et al. (2011) Histone tails regulate DNA methylation by allosterically activating de novo methyltransferase. Cell Res 21, 1172-1181 10.1038/cr.2011.92 [PubMed: 21606950]

58. Zhang Z-M, Lu R, Wang P, Yu Y, Chen D, Gao L et al. (2018) Structural basis for DNMT3Amediated de novo DNA methylation. Nature 554, 387-391 10.1038/nature25477 [PubMed: 29414941]

59. Yang L, Rau R and Goodell MA (2015) DNMT3A in haematological malignancies. Nat. Rev. Cancer 15, 152-165 10.1038/nrc3895 [PubMed: 25693834]

60. Emperle M, Rajavelu A, Kunert S, Arimondo PB, Reinhardt R, Jurkowska RZ et al. (2018) The DNMT3A R882H mutant displays altered flanking sequence preferences. Nucleic Acids Res 46, 3130-3139 10.1093/nar/gky168 [PubMed: 29518238]

61. Rošic S, Amouroux R, Requena CE, Gomes A, Emperle M, Beltran T et al. (2018) Evolutionary analysis indicates that DNA alkylation damage is a byproduct of cytosine DNA methyltransferase activity. Nat. Genet 50, 452-459 10.1038/s41588-018-0061-8 [PubMed: 29459678]

62. Jurkowska RZ, Rajavelu A, Anspach N, Urbanke C, Jankevicius G, Ragozin S et al. (2011) Oligomerization and binding of the Dnmt3a DNA methyltransferase to parallel DNA molecules: heterochromatic localization and role of Dnmt3L. J. Biol. Chem 286, 24200-24207 10.1074/ jbc.M111.254987 [PubMed: 21566127] 
63. Baubec T, Colombo DF, Wirbelauer C, Schmidt J, Burger L, Krebs AR et al. (2015) Genomic profiling of DNA methyltransferases reveals a role for DNMT3B in genic methylation. Nature 520, 243-247 10.1038/nature14176 [PubMed: 25607372]

64. Gahurova L, Tomizawa S. i., Smallwood SA, Stewart-Morgan KR, Saadeh H, Kim J et al. (2017) Transcription and chromatin determinants of de novo DNA methylation timing in oocytes. Epigenet. Chromatin 10, 25 10.1186/s13072-017-0133-5

65. Rajavelu A, Jurkowska RZ, Fritz J and Jeltsch A (2012) Function and disruption of DNA methyltransferase 3a cooperative DNA binding and nucleoprotein filament formation. Nucleic Acids Res 40, 569-580 10.1093/nar/gkr753 [PubMed: 21926161]

66. Emperle M, Rajavelu A, Reinhardt R, Jurkowska RZ and Jeltsch A (2014) Cooperative DNA binding and protein/DNA fiber formation increases the activity of the Dnmt3a DNA methyltransferase. J. Biol. Chem 289, 29602-29613 10.1074/jbc.M114.572032 [PubMed: 25147181]

67. Stepper P, Kungulovski G, Jurkowska RZ, Chandra T, Krueger F, Reinhardt R et al. (2017) Efficient targeted DNA methylation with chimeric dCas9-Dnmt3a-Dnmt3L methyltransferase. Nucleic Acids Res 45, 1703-1713 10.1093/nar/gkw1112 [PubMed: 27899645]

68. Norvil AB, Petell CJ, Alabdi L, Wu L, Rossie S and Gowher H (2018) Dnmt3b methylates DNA by a noncooperative mechanism, and its activity is unaffected by manipulations at the predicted dimer interface. Biochemistry 57, 4312-4324 10.1021/acs.biochem.6b00964 [PubMed: 27768276]

69. Gowher H and Jeltsch A (2002) Molecular enzymology of the catalytic domains of the Dnmt3a and Dnmt3b DNA methyltransferases. J. Biol. Chem 277, 20409-20414 10.1074/jbc.M202148200 [PubMed: 11919202]

70. Li J-Y, Pu M-T, Hirasawa R, Li B-Z, Huang Y-N, Zeng R et al. (2007) Synergistic function of DNA methyltransferases Dnmt3a and Dnmt3b in the methylation of Oct4 and Nanog. Mol. Cell. Biol 27, 8748-8759 10.1128/MCB.01380-07 [PubMed: 17938196]

71. Noh K-M, Wang H, Kim HR, Wenderski W, Fang F, Li CH et al. (2015) Engineering of a histonerecognition domain in Dnmt3a alters the epigenetic landscape and phenotypic features of mouse ESCs. Mol. Cell 59, 89-103 10.1016/j.molcel.2015.05.017 [PubMed: 26073541]

72. Petell CJ, Alabdi L, He M, San Miguel P, Rose R and Gowher H (2016) An epigenetic switch regulates de novo DNA methylation at a subset of pluripotency gene enhancers during embryonic stem cell differentiation. Nucleic Acids Res 44, 7605-7617 10.1093/nar/gkw426 [PubMed: 27179026]

73. Rondelet G, Dal Maso T, Willems L and Wouters J (2016) Structural basis for recognition of histone H3K36me3 nucleosome by human de novo DNA methyltransferases 3A and 3B. J. Struct. Biol 194, 357-367 10.1016/j.jsb.2016.03.013 [PubMed: 26993463]

74. Dhayalan A, Rajavelu A, Rathert P, Tamas R, Jurkowska RZ, Ragozin S et al. (2010) The Dnmt3a PWWP domain reads histone 3 lysine 36 trimethylation and guides DNA methylation. J. Biol. Chem 285, 26114-26120 10.1074/jbc.M109.089433 [PubMed: 20547484]

75. Neri F, Rapelli S, Krepelova A, Incarnato D, Parlato C, Basile G et al. (2017) Intragenic DNA methylation prevents spurious transcription initiation. Nature 543, 72-77 10.1038/nature21373 [PubMed: 28225755]

76. Wagner EJ and Carpenter PB (2012) Understanding the language of Lys36 methylation at histone H3. Nat. Rev. Mol. Cell Biol 13, 115-126 10.1038/nrm3274 [PubMed: 22266761]

77. Rinaldi L, Datta D, Serrat J, Morey L, Solanas G, Avgustinova A et al. (2016) Dnmt3a and Dnmt3b associate with enhancers to regulate human epidermal stem cell homeostasis. CellStemCell 19, 491-501 10.1016/j.stem.2016.06.020

78. Barau J, Teissandier A, Zamudio N, Roy S, Nalesso V, Hérault Y et al. (2016) The DNA methyltransferase DNMT3C protects male germ cells from transposon activity. Science 354, 909912 10.1126/science.aah5143 [PubMed: 27856912]

79. Jain D, Meydan C, Lange J, Claeys Bouuaert C, Lailler N, Mason CE et al. (2017) Rahu is a mutant allele of Dnmt3c, encoding a DNA methyltransferase homolog required for meiosis and transposon repression in the mouse male germline. PLoS Genet 13, e1006964 10.1371/ journal.pgen.1006964 [PubMed: 28854222] 
80. Chen T, Ueda Y, Xie S and Li E (2002) A novel Dnmt3a isoform produced from an alternative promoter localizes to euchromatin and its expression correlates with active de novo methylation. J. Biol. Chem 277, 38746-38754 10.1074/jbc.M205312200 [PubMed: 12138111]

81. Manzo M, Wirz J, Ambrosi C, Villaseñor R, Roschitzki B and Baubec T (2017) Isoform-specific localization of DNMT3A regulates DNA methylation fidelity at bivalent CpG islands. EMBO J 36, 3421-3434 10.15252/embj.201797038 [PubMed: 29074627]

82. Liao J, Karnik R, Gu H, Ziller MJ, Clement K, Tsankov AM et al. (2015) Targeted disruption of DNMT1, DNMT3A and DNMT3B in human embryonic stem cells. Nat. Genet 47, 469-478 10.1038/ng.3258 [PubMed: 25822089]

83. Gordon CA, Hartono SR and Chédin F (2013) Inactive DNMT3B splice variants modulate de novo DNA methylation. PLoS ONE 8, e69486 10.1371/journal.pone.0069486 [PubMed: 23894490]

84. Duymich CE, Charlet J, Yang X, Jones PA and Liang G (2016) DNMT3B isoforms without catalytic activity stimulate gene body methylation as accessory proteins in somatic cells. Nat. Commun 7, 11453 10.1038/ncomms11453 [PubMed: 27121154]

85. Arand J, Spieler D, Karius T, Branco MR, Meilinger D, Meissner A et al. (2012) In vivo control of CpG and non-CpG DNA methylation by DNA methyltransferases. PLoS Genet 8, e1002750 10.1371/journal.pgen.1002750 [PubMed: 22761581]

86. Kinde B, Gabel HW, Gilbert CS, Griffith EC and Greenberg ME (2015) Reading the unique DNA methylation landscape of the brain: non-CpG methylation, hydroxymethylation, and MeCP2. Proc. Natl Acad. Sci. U.S.A 112, 6800-6806 10.1073/pnas.1411269112 [PubMed: 25739960]

87. Kinde B, Wu DY, Greenberg ME and Gabel HW (2016) DNA methylation in the gene body influences MeCP2-mediated gene repression. Proc. Natl Acad. Sci. U.S.A 113, 15114-15119 10.1073/pnas.1618737114 [PubMed: 27965390]

88. Chen L, Chen K, Lavery LA, Baker SA, Shaw CA, Li W et al. (2015) MeCP2 binds to non-CG methylated DNA as neurons mature, influencing transcription and the timing of onset for Rett syndrome. Proc. Natl Acad. Sci. U.S.A 112, 5509-5514 10.1073/pnas.1505909112 [PubMed: 25870282] 

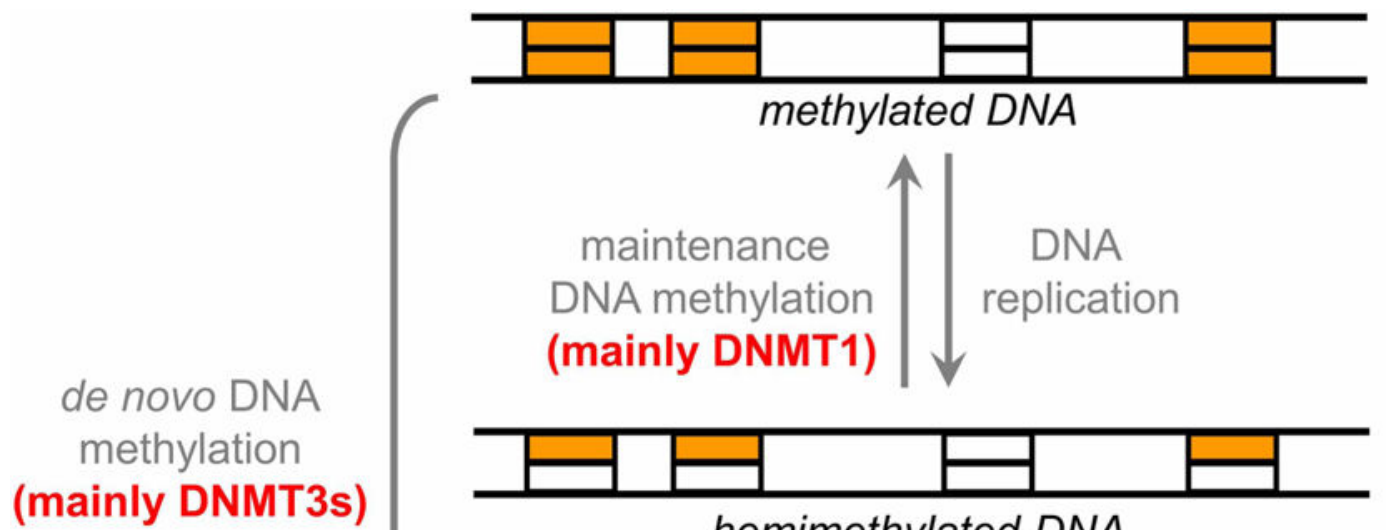

hemimethylated DNA

active DNA demethylation (TETs, TDG)

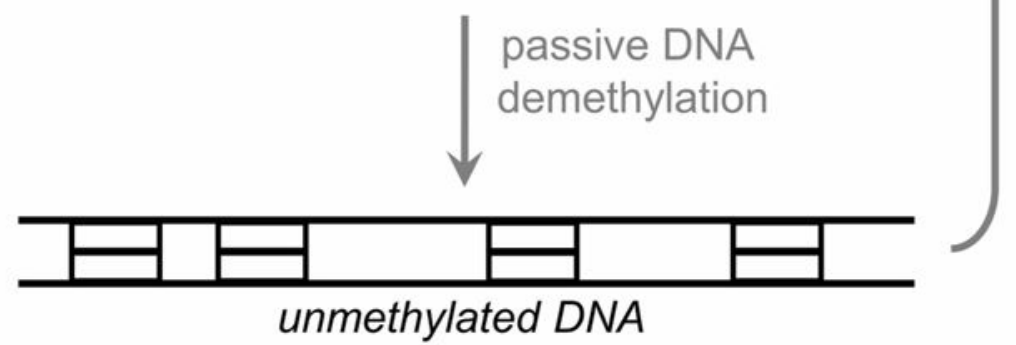

Figure 1. Cycle of DNA methylation in human cells and during development (adapted from [11]). DNA methylation patterns are generated during development and germ cell differentiation by de novo methyltransferases and kept through DNA replication by maintenance methylation. DNA methylation can be lost through passive or active demethylation. Typically, Dnmt1 is considered to be a maintenance enzyme, whereas Dnmt3a and Dnmt3b are regarded as de novo methyltransferases (TET, ten eleven translocation enzyme; TDG, thymine-DNA glycosylase). 


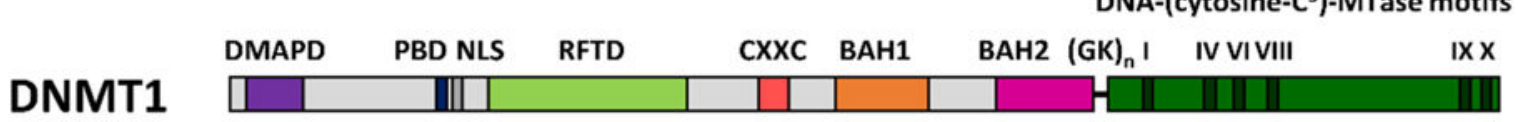

DNMT3A

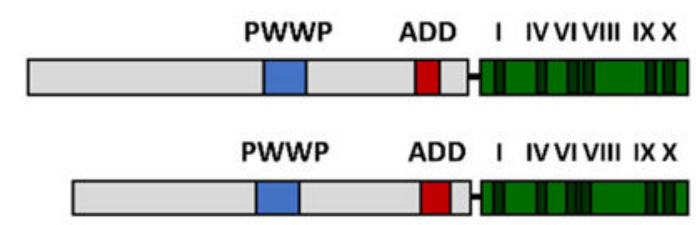

DNMT3B

DNMT3L

ADD I IV VIVIII

IDIIIIII

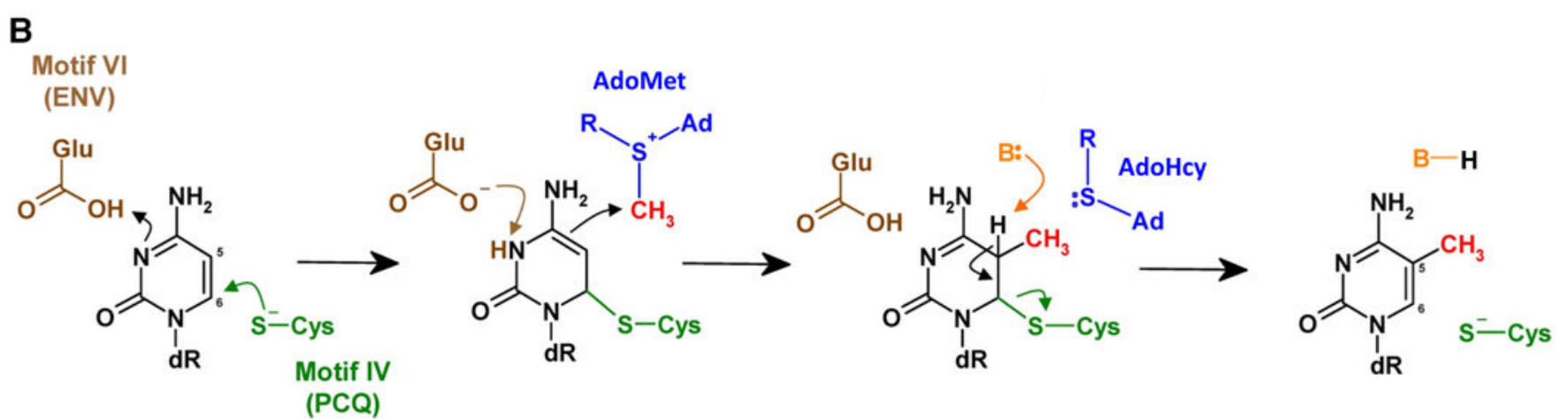

Figure 2. General features of DNMTs.

(A) Domain structure of the mammalian DNMT enzymes. The human DNMT1, DNMT3A, DNMT3B and DNMT3L proteins consist of 1616, 912, 853 and 387 amino acid residues, respectively. DMAPD, DNA methyltransferase-associated protein 1-interacting domain; PBD, PCNA-binding domain; NLS, nuclear localization signal; RFTD, replication foci targeting sequence (RFTS) domain; CXXC, CXXC domain; BAH1 and BAH2, bromoadjacent homology domains 1 and 2; $\mathrm{GK}_{\mathrm{n}}$, glycine lysine repeats; PWWP, PWWP domain; ADD, ATRX-DNMT3-DNMT3L domain (reprinted with permission from [6]). (B) Catalytic mechanism of cytosine C5 DNA methyltransferases (adapted from [12]). The catalytic glutamate residue from ENV motif (motif IV) is colored brown, the catalytic cysteine residue from PCQ motif (motif IV) is colored green, AdoMet is blue with the methyl group shown in red. The base for final proton abstraction (shown in orange) is not identified; it may be a water molecule. 

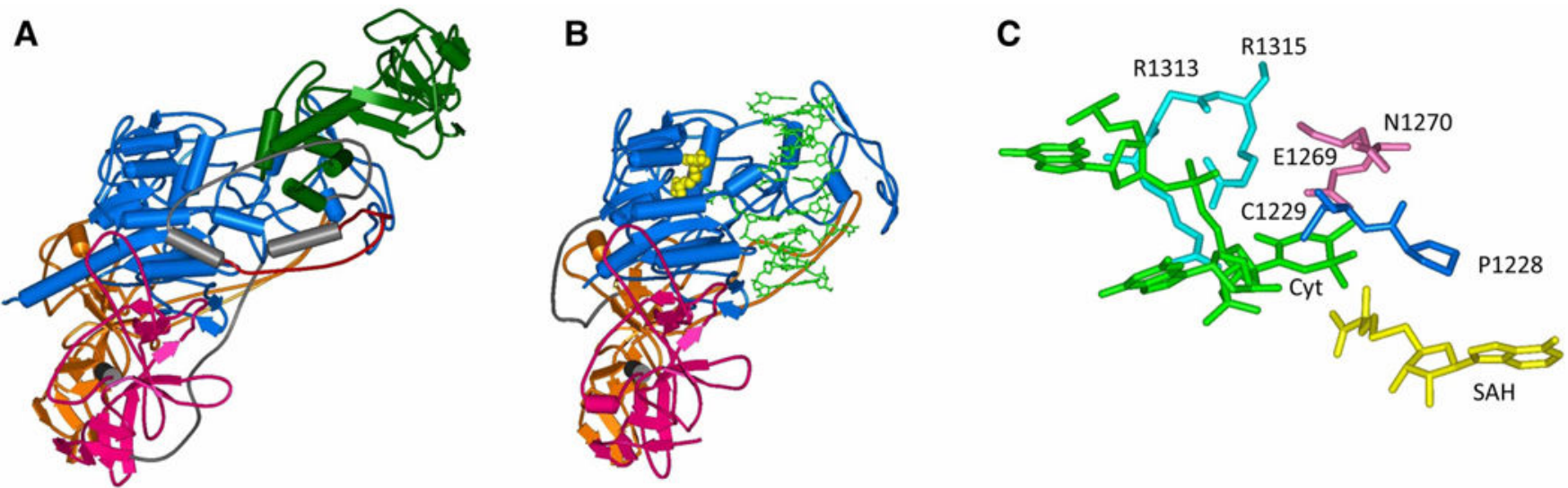

Figure 3. Structures of DNMT1.

(A) Structure of the DNMT1 apoenzyme [15] ( pdb 3AV4). The RFTS domain is shown in green, $\mathrm{CXXC}$ in red, BAH domains in orange and purple and the catalytic domain in blue. (B) Structure of a truncated C-terminal domain of DNMT in complex with hemimethylated DNA [21] ( pdb 4DA4). SAH is shown in yellow and DNA in light green. (C) Interaction of the flipped target base (Cyt) with the catalytic residues in the active site pocket of DNMT1 (C1339, E1369 and R1315). 

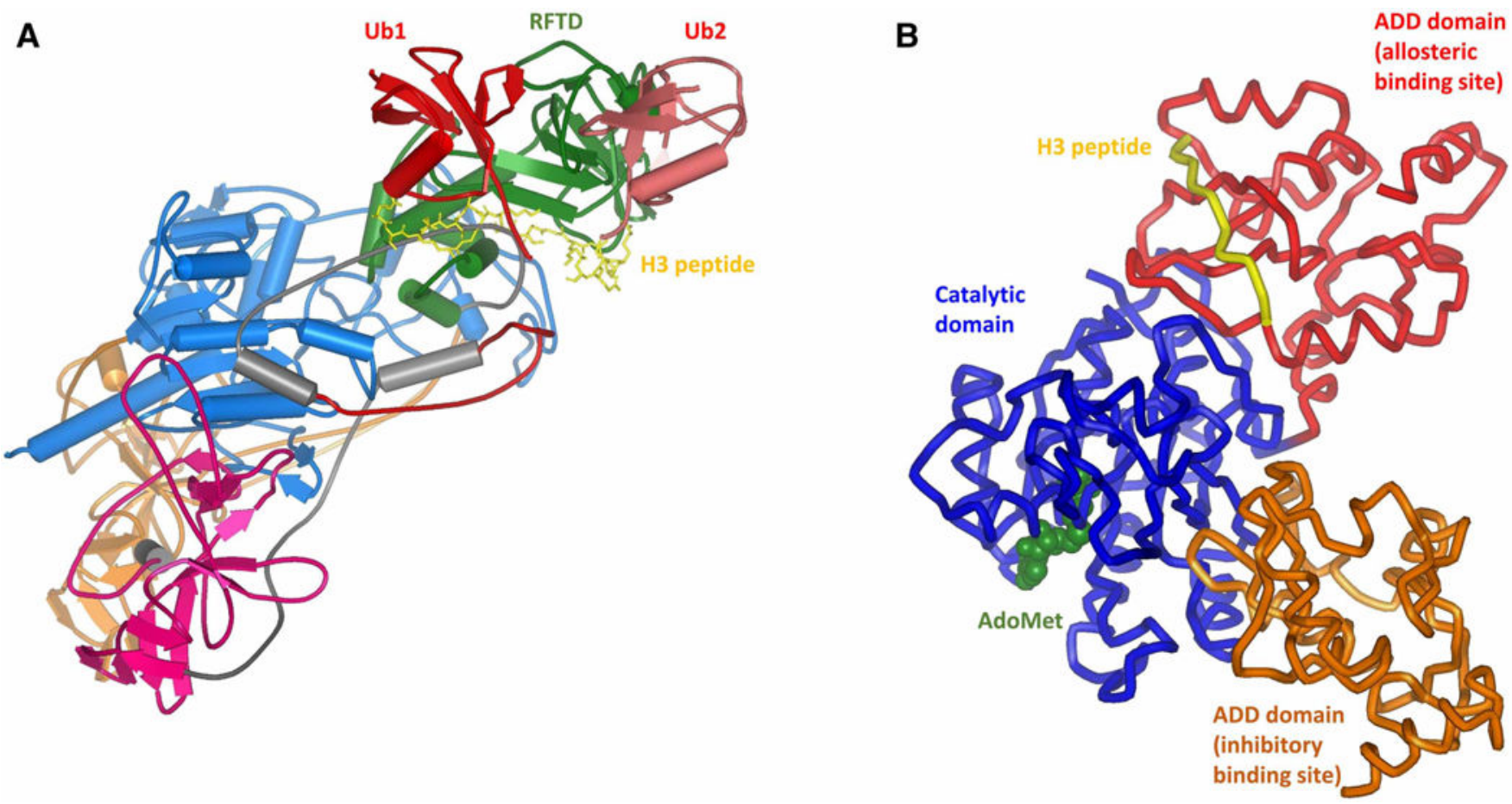

Figure 4. Interaction of DNMTs with chromatin modifications.

(A) Structure of the DNMT1 RFTS domain (green) in complex with the H3 peptide (shown in yellow) ubiquitinated at K18 and K23 [39] ( pdb 5WVO). The ubiquitin moieties are colored in red and light red. For illustration, the RFTS domain is overlaid with the structure of the DNMT1 apoenzyme colored and oriented as in Figure 3A. (B) Binding of the DNMT3A ADD domain to the catalytic DNMT3A domain (blue) in the DNMT3A/3L heterotetramer [55] ( pdb 4U7P and 4U7T). The ADD domain is shown in the autoinhibitory conformation (orange) and with bound peptide (yellow) in the catalytically active allosteric conformation (red). 


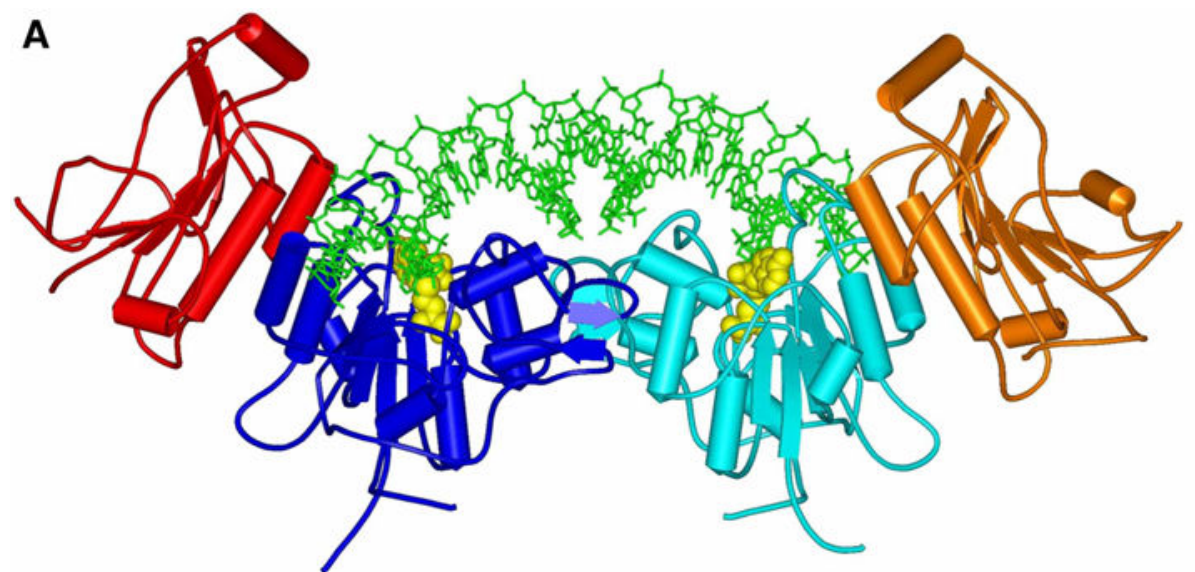

B

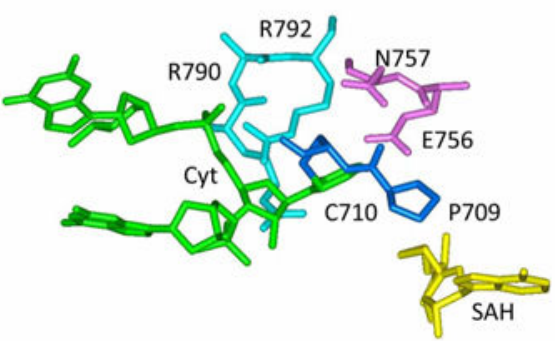

Figure 5. Structure of DNMT3A/3L in complex with DNA [58] ( pdb 6BRR).

(A) Overview of the structure. DNMT3A subunits are shown in blue and cyan, DNMT3L subunits in red and orange. SAH is shown in yellow, DNA in green. (B) Interaction of the flipped target base (Cyt) with the catalytic residues in the active site pocket of DNMT3A. 